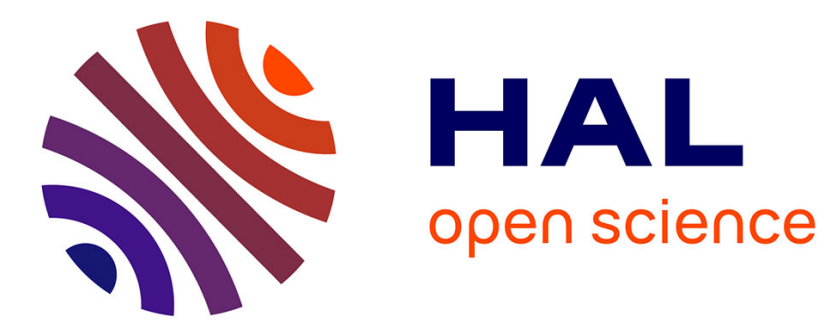

\title{
Le tournant entrepreneurial du droit du travail en France (1982-2017) \\ Laurent Willemez
}

\section{To cite this version:}

Laurent Willemez. Le tournant entrepreneurial du droit du travail en France (1982-2017) : Politiques néo-libérales, aggiornamento académique et espace de production d'un " sens commun ". L'Homme et la Société, 2021, Normes du droit du travail en France, 212, pp.169-194. 10.3917/lhs.212.0169 . halshs-03525304

\section{HAL Id: halshs-03525304 \\ https://shs.hal.science/halshs-03525304}

Submitted on 9 Feb 2022

HAL is a multi-disciplinary open access archive for the deposit and dissemination of scientific research documents, whether they are published or not. The documents may come from teaching and research institutions in France or abroad, or from public or private research centers.
L'archive ouverte pluridisciplinaire HAL, est destinée au dépôt et à la diffusion de documents scientifiques de niveau recherche, publiés ou non, émanant des établissements d'enseignement et de recherche français ou étrangers, des laboratoires publics ou privés. 
Paru dans L'homme et la société, n²12, janvier-juin 2020, p. 169-194

Le tournant entrepreneurial du droit du travail en France (1982-2017). Politiques néo-libérales, aggiornamento académique et espace de production d'un « sens

commun »

\author{
Laurent WILLEMEZ \\ Printemps ${ }^{1}$ (UMR_8085), Univ. Paris-Saclay/UVSQ, CNRS
}

Résumé

Les réformes du droit du travail en France dans les années 2016-2017 (loi El Khomri et ordonnances Macron) peuvent être vues comme l'acmé des politiques du travail menées depuis le milieu des années 1980. L'article revient sur cette véritable « révolution symbolique » que constitue le renversement du droit du travail d'un droit protecteur des salariés à un droit tourné vers l'entreprise. C'est le sens même du droit et sa signification qui ont donc changé, à la fois dans le monde du « social », entendu comme les acteurs politiques et administratifs des politiques du travail, et dans le champ académique, du côté des facultés de droit. Pour ce faire, l'article dresse d'abord un panorama des réformes mises en œuvre dans les trois dernières décennies et le discours qui les accompagne. Il analyse ensuite ses transformations analogues dans les facultés de droit et l'enseignement et de la recherche en droit du travail. Il revient enfin sur différents "lieux communs » où se croisent les différents acteurs de deux espaces sociaux, réunis autour de ce « tournant entrepreneurial » du droit du travail.

Mots-clés : droit du travail ; néo-libéralisme ; politiques du travail ; facultés de droit

The corporate side of French labour law and its victory (1982-2017). Neoliberal policies, turn in the academic field and production of a new common sense of labour law

\begin{abstract}
French labour laws' reforms in the last years can be considered in the continuity and the last step of the policy of work which have been pursued since the middle 80's. Thus, this article deals with the turn of labour law from a protective law of salaries into a law targeted at corporations, what can be seen as a "symbolic revolution". Such a change occurred as well in the political field (in the mind of politicians and administrators of policies of work) as in the academic field. To sketch it, I point first the reforms implemented since the last three decades. Then I analyse the way labour law is now taught in law schools, and has become a part of human resources' teaching. I finally stress how some places (commissions, ministers' offices, conferences...) become spaces where such a new sense of labour law has been built and maintained, through the meeting of actors of both fields.
\end{abstract}

Key words: labour law, neoliberalism, policies of work, law schools

\footnotetext{
${ }^{1}$ Professions Institutions Temporalités ; UFR des sciences sociales, 47, Boulevard Vauban, 78047 Guyancourt Cedex.
} 
Les lois El Khomri de 2016 puis les ordonnances Macron votées l'année suivante, qui transforment très profondément le droit du travail et son architecture consolidée dans les années 1970 et au début des années 1980 (entre les accords de Grenelle et les lois Auroux, pour résumer), n'apparaissent comme une nouveauté qu'aux yeux des observateurs les moins attentifs. Elles constituent en réalité le point culminant d'un processus de grande ampleur commencé dès 1986, et qui a réuni les gouvernements de droite comme de gauche autour d'un agenda de transformation radicale du droit du travail. Ce qui change pendant cette période, c'est la façon même de le penser: depuis sa création autour des années 1900, il a été considéré comme un cadre de protection des travailleurs et un ensemble de normes susceptibles d'amoindrir le déséquilibre entre le capital et le travail. Dans cette perspective, les élites sociales du début du $\mathrm{XX}^{\mathrm{e}}$ siècle, les «modernisateurs » des années 1930 et des années 1970, mais aussi les représentants politiques et syndicaux ouvriers tout au long du siècle défendent ensemble, malgré leurs désaccords radicaux, l'idée d'une « reconnaissance de la collectivité des salariés en tant qu'entité spécifique au sein même de l'entreprise. » (Le Goff, 2004 : 359). Ce qui conduit à introduire au sein de l'entreprise une représentation de ces salariés, représentation portant une parole particulière et des droits singuliers.

Tout change donc à partir du milieu des années 1980, qui marque une transformation profonde du « rapport salarial au cœur de l'évolution du capitalisme» (Amable \& Palombarini, 2018:111). Comme l'ensemble du langage politique, le discours sur la production et les salariés se modifie profondément en devenant un discours néo-libéral (Guilbert, Lebaron \& Peñafel, 2019), qui place en son cœur les profits de l'entreprise et sa rentabilité financière, présentés comme les seules conditions de possibilité de la réduction du chômage. Plus précisément encore, ces réformes sont néo-libérales dans la mesure où elles s'insèrent dans une idéologie conduisant à produire un cadre légal à même de garantir le libre fonctionnement du marché et de la concurrence. Il s'agit ce faisant de construire les conditions juridiques de possibilité de l'affaiblissement de l'État et de son pouvoir régulateur (Denord, 2016). Plus précisément, l'objectif est de remettre en cause les régulations liées au droit du travail, considérées comme autant de carcans étatiques et de normes trop nombreuses et trop lourdes empêchant la prise de risque et la flexibilité qui doivent être au cœur du gouvernement des travailleurs dans l'entreprise. La force de ce discours est qu'elle irrigue l'ensemble de la société et qu'elle se propage à tous les espaces de production du droit du travail (champ administratif, champ politique, champ académique). Elle semble par ailleurs devenir très rapidement un « discours sans adversaire » (Juhem, 2001).

C'est pour cette raison qu'on peut appeler cette transformation une « révolution symbolique» (Bourdieu, 2013), c'est-à-dire une révolution dans la manière même de penser le sens et la finalité du droit du travail et, in fine, d'imposer une nouvelle vision du monde dans l'ensemble de la société. Cette révolution symbolique cause donc un changement de la doxa elle-même, via des "lieux communs » qui la produisent : commissions parlementaires, rapports, think tanks, revues, colloques... Ce sont d'abord ces espaces, physiques ou symboliques, qu'il faut étudier pour comprendre les conditions de production et de diffusion de ce nouveau sens commun gouvernant la manière de penser le travail et ses régulations. Mais il faut aussi étudier les auteurs de ces productions, membres d'administrations centrales et de cabinets ministériels, experts du social, professeurs de droit du travail, syndicalistes et praticiens du droit. Il s'agit donc de faire l'hypothèse qu'il existe un espace social réunissant des agents issus de champs sociaux et d'espaces très différents, qui contribuent tous à produire un discours commun, traversant les habituels clivages politiques, disciplinaires ou sectoriels, autour de la nécessité de réformer le droit du travail au profit des entreprises et de la flexibilité qui serait nécessaire à leur profitabilité.

Cet article se propose de revenir sur ce discours devenu largement hégémonique, et sur les conditions et les acteurs de sa production tout au long des quelque quarante dernières années, en se focalisant plus particulièrement sur les réformes des années 2016 et 2017, qui en sont une illustration particulièrement aboutie. Pour ce faire, il s'agira en premier lieu de revenir sur ce discours de la réforme et la manière dont il a été produit et mis en application dans les politiques publiques de dérégulation du travail, avant de montrer comment le champ académique participe lui aussi de ce tournant entrepreneurial. Enfin, nous reviendrons sur un certain nombre de lieux où se croisent les différents producteurs de ce discours et de ces politiques. 


\section{Un droit du travail transformé}

\section{Il faut réformer le droit du travail : une doxa entre expertise économique et discours politiques}

Le discours émerge au début des années 1980 : il faudrait simplifier le droit du travail. Trop complexe, pas assez souple, trop tourné vers la sécurité des travailleurs et pas assez vers celle des entreprises, il serait par ailleurs trop lourd, trop étatique, pas assez flexible ni, dirait-on aujourd'hui dans le discours managérial, « agile ». Ce discours se retrouve perpétuellement, depuis le milieu des années 1980, dans la presse, dans les propos de nombreux professionnels de la politique ou d'essayistes, mais aussi dans l'ensemble des rapports, demandés par l'exécutif ou par des think tanks, rédigés entre 1983 et 2016 pour le dernier en date.

Une généalogie de ce discours dans le débat intellectuel trouverait sans doute ses origines dans un rapport que François Ewald rédige pour la Fondation Saint-Simon en 1983 : il y reproche au droit du travail de «s'enfler, de se compliquer » et d'être un domaine du droit où « les normes prolifèrent. » (Ewald, 1983) On peut rappeler que François Ewald, maoïste au début des années 1970, assistant de Michel Foucault au Collège de France, et en cela un de ses " exécuteurs testamentaires » après sa mort, a fait sa thèse sur les accidents du travail et la diffusion de la culture du risque dans le monde du travail à la fin du XIX ${ }^{\mathrm{e}}$ siècle (Ewald, 1986). Si l'on peut sans doute mesurer en quoi le tournant de Foucault vers l'analyse de la gouvernementalité aide Ewald à penser un État affaibli et le projet d'une société fondée sur la responsabilité (Behrent, 2010), il est néanmoins délicat de comprendre l'évolution de son parcours vers la Fédération française des sociétés d'assurance et le Medef. Quoi qu'il en soit, cette première mise en discussion de la nécessité d'alléger le droit du travail, un an après les lois Auroux, prend place dans une « Note »Note de la Fondation Saint-Simon (Ewald, 1983), structure qui réunit universitaires, essayistes, journalistes et chefs d'entreprises, aussi bien proches du parti socialiste que de l'opposition de droite de l'époque. On a là le modèle qui se développera au long des trente années suivantes, dans le rapprochement de ces élites et leur union autour d'un projet commun d'affaiblissement des normes juridiques encadrant le travail. Ces élites politico-intellectuelles sont aussi très largement pro-européennes, et de fait, comme Philippe Légé l'a montré, les différentes réformes françaises relatives au «dialogue social» et plus largement à l'ensemble du droit du travail ont partie liée avec les injonctions de la Commission européenne à lutter contre la "rigidité » du marché du travail français (Légé, 2019).

De fait, les rapports se suivent et se ressemblent: en 1995, le journaliste économique Jean Boissonnat préside un groupe de travail, initié par le Commissariat général au Plan et consacré au thème « Le travail et l'emploi en France, à l'horizon 2015 ». Le rapport qui s'en suivit, Le travail dans vingt $a n s^{2}$, propose une analyse prospective de l'évolution du travail et insiste, notamment dans sa préface, sur « le cadre institutionnel et légal du travail» et sa « reconstruction », qui apparait comme " la réforme clé pour mettre en œuvre une conception du travail dans les vingt prochaines années » (Boissonnat, 1995 : 29-30). Sur ce plan, le rapport s'appuie sur un document interne intitulé « Droit au travail et régulation des rapports sociaux » rédigé par Thierry Priestley, directeur d'administration centrale au ministère du Travail. Le rapport final insiste sur «l'opérationnalité déclinante du droit du travail » et les «dysfonctionnements » de celui-ci, notamment en ce qui concerne le temps de travail (ibid. : 89-90). Il est enfin «confronté à deux difficultés majeures d'adaptation aux évolutions en cours » : les « mutations que connaît notre système productif» et « le poids historique de l'État dans la régulation des rapports de travail. » (ibid.: 92). Les conséquences en sont claires : il faut créer un contrat de travail plus flexible et il faut décentraliser la négociation en la rapprochant de l'entreprise.

Les années 2000 voient la multiplication de ces rapports ${ }^{3}$, qui ne sont, bien sûr, plus demandés par le Commissariat général au Plan, mais plutôt par des premiers ministres ou des ministres du travail voulant préparer des réformes, ou à d'autres moments par des organisations patronales ou des think tanks. Ces rapports sont précédés de «lettres de mission» qui toutes présentent le droit du travail comme «trop sophistiqué » et "contradictoire», " peu intelligible et sans cohérence», trop « incertain » et pas assez « lisible » ni « accessible », résultat d'une "prolifération » de règles trop

\footnotetext{
${ }^{2}$ Ce rapport a fait l'objet d'une publication. Voir Boissonnat, 1995.

${ }^{3}$ Plusieurs de ces rapports sont listés dans l'encadré 1, p. 5.
} 
uniformes. Le constat est toujours le même : si le travail et l'emploi sont en crise, c'est parce que le droit du travail crée des rigidités trop importantes. Il faut donc le « réformer » dans son ensemble, que ce soit dans son aspect individuel, autour du contrat de travail, ou collectif, autour de la place des organisations syndicales dans l'entreprise.

Tous ces écrits se ressemblent étrangement, en particulier parce qu'ils prennent comme point de départ « la force de l'évidence » néo-libérale (Guilbert, 2008), largement diffusée par l'économie du travail néo-classique. L'exemple le plus paradigmatique de ce modèle est sans doute le rapport de Jacques Barthélémy et Gilbert Cette (2015), qui explicitent leur démarche au début de l'ouvrage qui en est tiré :

\begin{abstract}
«Une abondante littérature s'est efforcée de caractériser les effets des rigidités règlementaires concernant le marché du travail sur la dynamique des entreprises et l'équilibre macro-économique. Les indicateurs mobilisés pour caractériser les rigidités du droit social sont variés, mais les enseignements de ces analyses sont convergents. Un droit social règlementaire important influence négativement l'équilibre sur le marché du travail et la productivité, ces deux types d'effets n'étant bien entendu pas indépendants. » (Barthélémy \& Cette, 2015 : 30)
\end{abstract}

Il faudrait réinscrire ces prises de position dans l'histoire des sciences économiques - et plus particulièrement dans l'économie du travail - depuis la fin des années 1970 pour revenir sur la manière dont s'est diffusée la théorie orthodoxe, fondée sur des modèles économétriques, permettant de montrer combien les « rigidités » créées par les institutions et les régulations juridiques françaises conduisent à produire du chômage et à mettre en danger la compétitivité des entreprises françaises. Ce courant tient une place importante en France depuis le début des années 2000, et Francis Kramarz, ainsi que ses collègues du CREST ${ }^{4}$ peuvent être considérés, dans une certaine mesure, comme des exemples paradigmatiques d'économistes qui construisent des modèles économétriques formalisés mettant en relations les indicateurs liés aux structures juridiques d'encadrement du marché du travail, les coûts pour les entreprises et les résultats en termes d'emplois et de croissance économique. Cette expertise économique est ensuite mise à profit dans des rapports ministériels, dont par exemple celui de 2004, rédigé par Pierre Cahuc et Francis Kramarz à la demande de Nicolas Sarkozy, ministre de l'Économie, et de Jean-Louis Borloo, ministre de l'Emploi, qui a pour objet de défendre la mise en place d'une « sécurité sociale professionnelle » et se termine par la nécessité d' « unifier le contrat de travail », autrement dit de supprimer le contrat à durée indéterminée (CDI) (Cahuc \& Kramarz, 2004).

Ces discours sur la nécessité de la réforme sont tout à fait consonants avec des prises de positions entendues pour d'autres domaines d'action publique, qu'il s'agisse de la réforme des services publics (université, justice, santé), plus largement des différents aspects du rapport salarial, et même de la société dans son ensemble. On reconnaît là ce que Pierre Dardot et Christian Laval appellent «la nouvelle raison du monde ", et qui consiste à étendre à de nombreux domaines de la vie sociale les logiques de l'efficience et de la rentabilité fondant le système capitaliste financiarisé actuel (Dardot \& Laval, 2009). À chaque fois, il s'agit de mettre en œuvre un discours présentant une institution ou un domaine comme «en crise », ce qui nécessiterait des réformes permettant de les moderniser. Plus précisément, concernant le monde du travail proprement dit, ces prises de position dans le champ académique et celui de l'expertise renvoient aux analyses consacrées aux transformations du rapport salarial, comme l'indiquent Amable et Palombarini: "Le thème de la flexibilité externe s'est lentement imposé comme le mode dominant de transformation du rapport salarial, supposée garantir des ajustements rapides et efficaces de la main-d'œuvre vers les secteurs et les firmes les plus efficaces. (Amable \& Palombarini 2018: 113). C'est cet environnement tout à la fois intellectuel, expert et politique qui sous-tend les mesures prises depuis le milieu des années 1980.

\footnotetext{
${ }^{4}$ Le CREST (Centre de recherche en économie et statistique) est le laboratoire CNRS d'économie (et, sur un
} plan très minoritaire, de sociologie) de l'ENSAE et de l'École polytechnique. 


Encadré 1 : Les principaux rapports (et lettres de mission) appelant à réformer le droit du
travail, par ordre chronologique
LANGLOIS Philippe, 2004. Réflexions sur la simplification du droit du travail, Institut de l'entreprise.
VIRVILLE Michel de, 2004. Pour un code du travail plus efficace. Rapport au ministre des Affaires
sociales, du Travail et de la Solidarité, Paris, La Documentation française.
CAHUC Pierre \& KRAMARZ Francis, 2004. De la précarité à la mobilité : vers une sécurité sociale
professionnelle: rapport au Ministre de l'économie, des finances et de l'industrie et au Ministre de
l'emploi, du travail et de la cohésion sociale, Paris, La Documentation française.
HADAS-LEBEL Raphaël, 2006. Pour un dialogue social efficace et légitime : représentativité et
financement des organisations professionnelles et syndicales, Rapport au Premier ministre.
Lettre de mission du ministre Xavier Darcos pour la « simplification du droit du travail », 2010.
BARTHELEMY Jacques \& CETTE Gilbert, Réformer le droit du travail (rapport pour le think tank Terra
Nova) Paris, Odile-Jacob, 2015.
COMBREXELLE Jean-Denis, 2015. La négociation collective, le travail et l'emploi, Rapport au Premier
ministre.
BADINTER Robert, 2016. Rapport au Premier ministre du Comité chargé de définir les principes
essentiels du droit du travail.

Les mesures mises en æuvre

C'est donc l'ensemble de l'architecture du droit du travail comme cadre de protection des salariés et de réduction des inégalités entre ceux-ci et l'employeur qui est attaqué tout au long des trente dernières années (avec une pause entre 1997 et 2002) ; attaque dont l'acmé se situe sans conteste dans les années 2016-2017. La transformation de la conjoncture intellectuelle, dont nous avons repéré l'origine au début des années $1980^{5}$, trouve son pendant dans le basculement des politiques économiques et sociales françaises. Ce dernier s'opère au moment du choix, par François Mitterrand, de privilégier une politique monétaire conforme aux exigences de la construction européenne et, donc, de privilégier une politique économique fondée notamment sur l'obligation de faire baisser les dépenses publiques, ainsi que sur la nécessité d'accroître la compétitivité des entreprises et de privilégier leur bonne santé financière. Les politiques publiques sont alors mises en œuvre dans le respect de ces nouvelles contraintes, et les politiques du travail n'échappent pas à ce tournant entrepreneurial et de déréglementation. Dans ces conditions, la baisse de la durée du travail à 39h et l'obtention de la cinquième semaine de congés payés en 1981, mais surtout les lois Auroux - qui ont été pensées comme permettant la création d'une nouvelle architecture pour le droit du travail et une manière de « changer le travail » en accroissant « les droits des travailleurs $\rangle^{6}$ (Tracol, 2009) - peuvent être considérées comme les derniers feux d'une "conception offensive du droit du travail» (Willemez, 2017 : chap. 3).

À partir de 1986 et de la loi supprimant l'autorisation administrative de licenciement, les réformes se multiplient dans cette direction unique. Elles touchent l'ensemble du dispositif de protection qui avait été construit depuis le début $\mathrm{du} \mathrm{XX}^{\mathrm{e}}$ siècle, que ce soit le contrat de travail et la possibilité de sa rupture, ou le dialogue social et la place des représentants des travailleurs dans les décisions de l'entreprise les concernant. Ainsi, les possibilités de contrats "atypiques» se multiplient et sont légalisées (institutionnalisation du $\mathrm{CDD}$, accroissement de la possibilité de recourir à l'intérim, création des CDI à durée restreinte - contrats de chantier, par exemple). Toujours pour ce qui concerne le contrat de travail, les trente dernières années ont surtout rendu plus facile sa rupture, par le licenciement ou l'accord interindividuel (via la rupture conventionnelle). La catégorie de licenciement économique, créée en 1975 au moment du début de la crise économique et sociale, a donné lieu à un travail normatif important, autour d'un accroissement de la souplesse dans son utilisation. Les " plans sociaux », devenus d'une manière encore plus euphémisée " plans de sauvegarde de l'emploi » à partir de 2002, permettent ainsi à l'entreprise de multiplier les manières de supprimer des emplois (aux

\footnotetext{
${ }^{5}$ Mais qui pourrait sans doute démarrer encore plus tôt, au moment de l'essor du néo-libéralisme en France au milieu des années 1970. Voir Denord, 2016.

${ }^{6}$ C'était d'ailleurs le titre du rapport de septembre 1981, prélude à l'élaboration des lois Auroux.
} 
« licenciements secs » s'adjoignent des mesures collectives de reconversion, de reclassement ou de départ à la retraite), quand celle-ci est en difficulté. C'est d'ailleurs cette mesure de la difficulté économique des entreprises qui est rediscutée tout au long de la période, ses indicateurs devenant de moins en moins précis et de plus en plus élastiques. Sur le plan des licenciements individuels, liés à des fautes du salarié, on note là-encore un assouplissement des règles juridiques. Dans ce cas, ce n'est pas la loi relative au licenciement pour faute (datant de 1973) qui a été réformée, mais un ensemble de mesures ont contribué à en amoindrir largement l'effet. En 2008 est instaurée la rupture conventionnelle, qui permet aux deux parties de se mettre d'accord sur une rupture du contrat de travail - ce qui, en réalité, rend possible un licenciement à faible coût. En 2014, la loi Macron «pour la croissance, l'activité et l'égalité des chances économiques » fragilise l'institution prud'homale, précisément chargée d'assurer le respect des règles légales de ce mode de licenciement. Cette première attaque contre les conseils de prud'hommes n'est rien à côté de celle qui viendra en 2017, mais c'est déjà la logique même de régulation juridique et judiciaire du licenciement qui est mise en cause.

Cependant le bouleversement le plus important concerne peut-être la question de la place de la représentation, notamment syndicale, des salariés dans la production des règles d'encadrement du travail. Le sens général de la période est celui d'une primauté croissante du « dialogue social » sur la logique étatique et en même temps de l'abaissement vers l'entreprise du niveau pertinent de production de l'accord entre les «partenaires sociaux». Si les sociologues des relations professionnelles ont largement documenté ces profondes transformations (Mias et al., 2016), il est cependant intéressant de retracer rapidement leur histoire. Les lois Auroux rendent obligatoire la négociation collective dans l'entreprise, mais c'est un accord national interprofessionnel en 1995, puis une «Déclaration commune » des syndicats (non signée par la seule CGT) qui élargissent le champ des accords d'entreprise. La loi Fillon de 2004 autorise les accords d'entreprise à déroger aux accords de branche et supprime la règle de faveur ${ }^{7}$. La loi d'août 2008 " réformant la démocratie sociale » fait de la convention de branche ou de l'accord d'entreprise les outils légitimes de réglementation en termes de temps de travail (ce qui rend donc désormais inutile de légiférer sur ces questions) et transforme profondément les règles de représentativité des organisations syndicales. Enfin, la loi Rebsamen de 2015 permet de créer dans les entreprises de plus de 300 salariés une institution unique de délégation du personnel réunissant délégués du personnel, comités d'entreprises et comités d'hygiène, de sécurité et des conditions de travail (CHSCT).

Même si ce mouvement de réforme existe depuis les années 1980, on note une accélération à partir du milieu des années 2000, accélération qui est à mettre au crédit aussi bien de gouvernements de droite que de ceux issus du parti socialiste. Dans ce cadre, les deux derniers dispositifs législatifs synthétisent et prolongent l'ensemble de ces transformations. La première est la Loi El Khomri, du nom de la ministre socialiste du travail de la présidence Hollande, votée en 2016 ; la seconde est un ensemble de lois, prises par ordonnances dans les premiers mois de la présidence d'Emmanuel Macron, en 2017. Bruno Amable et Stefano Palombarini montrent très clairement dans quelle mesure on peut dresser une continuité évidente entre les dernières présidences dans la défense d'une nouvelle " alliance sociale », qu'ils appellent « bloc bourgeois », et qui réunit les anciens adversaires politiques qui étaient le parti socialiste et les partis de droite autour d'une volonté de "réformer» le modèle social français. Et dans ce cadre, un des objectifs phares est bien la "poursuite de la réforme de la relation salariale» (Amable \& Palombarini, 2018:176). Parmi la multiplicité des mesures, la principale est sans doute le basculement complet de la hiérarchie des normes, les accords d'entreprise étant prioritaires par rapport aux accords de branches, et la loi réduite à un « rôle résiduel » (Amable \& Palombarini, 2018 : 180). Par ailleurs, sur ce même plan de la place des représentants du personnel, le Comité social et économique (CSE) vient fusionner en une seule structure le comité d'entreprise, les délégués du personnel et les CHSCT. Mais d'autres mesures touchent aussi le contrat de travail et sa rupture, tels le rétrécissement du périmètre géographique (la France et non plus l'ensemble du groupe) pour apprécier les difficultés économiques des entreprises dans le cas de licenciements collectifs ; la fin de l'obligation de motiver le licenciement individuel dans la lettre de licenciement adressée au salarié ; la possibilité de réaliser des ruptures conventionnelles collectives ; ou encore l'affaiblissement

\footnotetext{
${ }^{7}$ Cette règle faisait que ni l'accord d'entreprise ni l'accord de branche ne pouvaient déroger à la loi si ceux-ci étaient moins favorables que celle-là aux salariés.
} 
radical des prud'hommes (instauration de plafonds d'indemnité en cas de condamnation de l'employeur et raccourcissement des délais permettant la saisine), etc.

On voit donc comment l'ensemble du programme néo-libéral prôné par les économistes et juristes du travail dans leurs différents rapports est mis en œuvre, dans une logique très clairement affirmée de remise en cause radicale du travail institué, pour reprendre la belle formule de Claude Didry (2016). L'expression pourrait réunir toutes les structures d'encadrement du monde du travail et tous les espaces de régulation formelle, en l'occurrence juridique, de celui-ci. On pourrait montrer ainsi que dans le projet de ces réformateurs - dont la réussite finale n'est certes pas assurée mais dont l'avancée est réelle -, la seule institution du travail qui vaille est la direction de l'entreprise, qui impose ses choix, définit ses interlocuteurs et met en œuvre seule ses objectifs financiers.

\section{Consolidation, « gestionnarisation » et polarisation du droit du travail}

Le tournant entrepreneurial du droit du travail est donc d'abord le résultat d'un projet politique, appuyé sur l'expertise d'économistes mais aussi de juristes. On croise souvent, parmi les auteurs des rapports et les membres des commissions, des professeurs de droit du travail qui défendent eux aussi cette vision d'un droit du travail tourné vers l'entreprise et sa rentabilité plutôt que vers le salarié et sa protection. Il s'agit maintenant de dessiner les contours de cette transformation du droit du travail académique.

\section{Un domaine du droit institutionnalisé}

À la fin des années 1970, le droit du travail est désormais une discipline établie dans les facultés de droit. Elle a ses enseignants-chercheurs, ses doctorants, ses traités et manuels, ses revues et ses espaces de rencontre, qu'il s'agisse de colloques ou de "mélanges » célébrant le départ en retraite de professeurs et matérialisant les réseaux dont ceux-ci sont le centre. Il reste que cette "matière », comme on dit dans les facultés de droit, n'est pas dominante, comme le montre la faible présence des travaillistes (pour reprendre le terme indigène) dans les jurys d'agrégation, ou plus largement l'impossible émancipation du droit social (ou droit du travail) par rapport au droit privé. Quoi qu'il en soit, la période qui suit est celle de la consolidation de ce domaine du droit.

Cette consolidation passe néanmoins par la persistance de quelques foyers historiques de présence forte du droit du travail et de la place importante occupée par un certain nombre d'acteurs centraux et dominants, professeurs et directeurs de thèse, ayant fait « école » auprès de leurs étudiants, eux-mêmes devenus parfois enseignants-chercheurs dans les établissements de leurs "patrons de thèse ". La plupart de ces personnages centraux sont par ailleurs des hommes. Sans être exhaustifs, et sans prétention d'établir un quelconque palmarès ${ }^{8}$, on peut repérer quelques-uns de ces foyers : Nanterre, Paris 1, Paris 2, Montpellier, Lyon 2, Strasbourg, Bordeaux, Nancy... Ces lieux ont souvent été construits par ce que l'on pourrait appeler des «pères fondateurs » du droit du travail (François de Menthon et Pierre-Henri Teitgen à Nancy, Jean-Maurice Verdier à Nanterre, Gérard Lyon-Caen à Paris $1 \ldots)$, souvent célébrés et dont l'héritage, qui prend la forme de la constitution d'une «tradition » ou d'une " école » (Chapoulie, 2001), devient une ressource stratégique dans les luttes académiques au sein du champ'.

Dans l'ensemble de ces lieux, on trouve une équipe constituée de droit social, réunie autour d'un centre de recherche (ou d'une équipe quand les universités ont souhaité avoir des laboratoires de taille conséquente) et d'un master comprenant plusieurs parcours, en formation initiale, par apprentissage ou en formation continue. ${ }^{10}$

\footnotetext{
${ }^{8}$ On peut d'ailleurs noter qu'aujourd'hui, ces centres universitaires et leurs masters sont objectivement en concurrence, dans des palmarès ou des benchmarks (réalisés par des médias ou par des sites spécialisés, filiales françaises d'entreprises de notation des formations).

${ }^{9}$ Il y a là un programme de recherche très riche pour poursuivre l'analyse, par exemple par une analyse en termes de réseaux.

${ }^{10}$ Il faut noter l'existence de deux laboratoires historiquement spécialisés dans le droit social et dont le CNRS est tutelle : le COMPTRASEC à Bordeaux et Droit et changement social à Nantes. Par ailleurs, deux autres
} 
Dans ce cadre général, un focus sur les masters permet de montrer ce qu'est aujourd'hui avant tout le droit social : un espace de formation de juristes d'entreprises et d'avocats tournés vers la gestion et vers l'entreprise.

Dans cette perspective, les diplômes spécialisés dits de «troisième cycle » avant la réforme LMD de 2004, à savoir des diplômes de bac +5 , se multiplient : plusieurs DEA sont créés dans les années 1980, puis des DESS, bac +5 à finalité professionnelle, dans les années 1990. Comme pour l'ensemble des disciplines, ces derniers répondent à la préoccupation croissante, dans l'enseignement supérieur, d'insertion des étudiants sur le marché du travail, mais aussi à l'essor de la profession d'avocat et, en son sein, de la spécialité en droit social, largement tournée vers le monde des entreprises. Les DEA et les DESS ont aujourd'hui largement fusionné dans des masters ayant à la fois une vocation professionnalisante et une voie orientée vers la recherche, comme dans la plupart des disciplines universitaires. Les dénominations de ces masters sont nombreuses, depuis le simple «droit social» jusqu'à «droit social et gestion des ressources humaines », en passant par «juriste de droit social » etc. Pourtant, tous se ressemblent a priori, dans leur orientation vers les métiers du droit des ressources humaines en général, qu'il s'agisse de postes dans les DRH des entreprises ou de collaborateurs, voire de salariés, dans les cabinets d'avocats (qu'ils aient une spécialité unique en droit social ou que celle-ci soit un de leurs multiples domaines d'activité). Ces masters ont aussi globalement le même type d'organisation, autour d'enseignements fondamentaux de droit du travail individuel et collectif, de cours de gestion des relations de travail en entreprise (droit des entreprises en difficultés, techniques de gestion prévisionnelle des emplois et compétences - GPEC - etc.). Certains masters vont même au-delà et proposent aussi en complément des cours de droit des affaires ou de droit fiscal. Les présentations de ces masters sur les sites internet sont assez révélatrices de l'orientation gestionnaire ou entrepreneuriale de leurs formations.

Ainsi tel master donne pour la formation les objectifs suivants :

« - assurer aux étudiants un début de spécialisation dans le domaine du droit social ;

- donner aux étudiants une formation qui intègre les dimensions européennes, internationales et comparatives du champ disciplinaire analysé ;

- donner aux étudiants les connaissances nécessaires à l'exercice des fonctions de juriste d'entreprise ou d'avocat spécialisé en droit social (qui constitue un secteur professionnel en pleine expansion) tout en leur apportant un complément de connaissances fondamentales (notamment en droit civil et en droit des affaires) de nature à permettre d'éventuelles réorientations. $\gg^{11}$

Tel autre, intitulé «droit social et GRH », est, comme son nom l'indique, résolument tourné vers la gestion des ressources humaines :

« le Master 2 DS-GRH est né d'un constat, celui de la nécessité d'allier les compétences du juriste à celles du responsable des ressources humaines pour apporter une réponse aux besoins des entreprises. Pour s'en convaincre, il suffit de penser à des problématiques récentes telles que la gestion prévisionnelle des emplois et des compétences, la prévention des risques psychosociaux ou la promotion de la diversité en entreprise. En conséquence et pour atteindre cet objectif, le Master 2 DSGRH propose à ses étudiants une formation d'excellence scientifique ouverte sur le monde professionnel. $\rangle^{12}$

laboratoires CNRS ont des équipes de droit social constituées : l'unité Droit, religion, entreprises et société (DRES) à Strasbourg et le Centre de recherches critiques sur le droit (CERCRID) à Lyon et Saint-Étienne.

${ }^{11}$ Ces objectifs sont issus de la présentation de la formation en Master 1 de droit social de l'Université Paris 2. Voir le site : https://www.u-paris2.fr/fr/formations/offre-de-formation/masters/master-1-droit-mention-droitsocial. (consulté le 28/11/2020).

12 Présentation du Master DS-GRH tenu à l'Université d'Orléans. Voir le site: http://www.m2dsgrh.fr/. (consulté le 28/11/2020). 
Ces transformations ne sont pas seulement internes au droit du travail, dans la mesure où elles renvoient plus largement aux changements structurels de l'enseignement universitaire, marqué par « un développement sans précédent du secteur professionnel» (Charle \& Soulié, 2007: 175). Cette professionnalisation croissante, qui peut, selon les disciplines, aller de pair avec l'affaiblissement de la recherche, est d'autant plus forte ici que les facultés de droit ont toujours appartenu, avec les facultés de médecine, au pôle le plus hétéronome du monde académique (Bourdieu, 1984), c'est-à-dire ici le plus sensible à la demande venue du monde des entreprises. De la même manière, on peut insister sur la diffusion des enseignements liés à la gestion dans nombre de cursus et au processus global de rapprochement entre formation en droit et formation en gestion (Biland, 2013). Pour autant, on voit bien la porosité potentiellement forte entre droit du travail et gestion, autour de l'espace des ressources humaines, dans les cabinets de conseil ou dans les grandes entreprises - là où l'emploi d'individus issus de masters de droit social est particulièrement important. Si l'on compare avec la période précédente, ce sont d'autres formes d'insertion professionnelle qui sont aujourd'hui fortement minorées (même si elles existent encore parfois) : les agents des directions du travail (notamment les inspecteurs du travail), les juristes d'organisations syndicales ou d'associations, ou encore les avocats spécialisés dans la défense des salariés et des syndicats.

\section{Un droit du travail de plus en plus polarisé}

Ce n'est pas seulement la description des formations qui permet de mettre l'accent sur ce tournant entrepreneurial du droit du travail, mais aussi l'activité même des professeurs de droit. Les années 1970 étaient marquées par la domination forte, au sein de ce champ académique particulier, d'un droit tourné vers la défense et la protection des salariés, autour d'un certain nombre de figures et de lieux que l'on peut citer sans souci d'exhaustivité : à Paris 1 avec Gérard Lyon-Caen, à Nanterre avec JeanMaurice Verdier, à Lyon avec Jean Pélissier et Antoine Jeammaud, ou encore à Strasbourg avec Hélène Sinay. Les trente dernières années marquent alors un véritable retournement avec la domination croissante d'un droit orienté vers l'entreprise, sa compétitivité et sa "sécurité juridique », pour reprendre les termes qui fleurissent sur ce plan. Le droit du travail à destination de la défense des salariés existe certes encore, mais il devient fortement minoritaire.

La lecture des écrits académiques montre l'étendue et la force de ce tropisme entrepreneurial, et par conséquent de ce véritable changement de paradigme, qui conduit à faire du droit du travail un droit de l'entreprise, voire un droit économique. Ainsi, dans l'introduction qu'il donne du colloque de Droit social de 2004, Bernard Teyssié annonce « le glissement du droit du travail dans la sphère du droit des affaires ». Il ajoute d'ailleurs que «l'exigence de compétitivité pénètre dans la sphère du droit du travail : nul ne peut y demeurer indifférent sauf à donner le sentiment de se désintéresser, par-delà les mots, du sort de l'entreprise donc, en définitive, de l'emploi. » (Teyssié, 2005 : 127). L'argument est imparable, et il est exactement le même, écrit avec le supplément d'élégance qui peut caractériser la langue juridique, que ce que l'on trouve dans le champ politique à la même époque - et jusqu'à aujourd'hui. Il peut être intéressant de revenir sur la figure de son auteur pour mieux comprendre le tournant qu'a pris le droit du travail. Celui-ci apparaît incontestablement, depuis le milieu des années 1980, comme un des acteurs dominants du droit du travail : né en 1948, auteur d'une thèse de droit commercial à l'Université de Montpellier 1, il publie dès 1980 son Traité de droit du travail puis, en 1992, un manuel en deux tomes aux éditions Litec, qui est encore mis à jour en 2018 (c'est sa onzième édition). Il publie de nombreux autres manuels (droit civil, droit des personnes, droit social européen...). Il cumule par ailleurs les positions de pouvoir au sein du champ académique : directeur scientifique du Jurisclasseur périodique « social », président du jury d'agrégation de droit privé et sciences criminelles en 2008, directeur du master professionnel « Droit et pratiques des relations de travail » à Assas. Il a enfin été président de l'Université Paris 2 Assas de 1997 à 2002, puis du Conseil national du droit de 2008 à 2010. Il a dirigé et dirige encore de très nombreuses thèses, ce qui fait de lui le leader d'une « école » de travaillistes proches de l'entreprise, qui aurait pour colonne vertébrale un axe reliant Paris 2 à Montpellier 1, dont l'acteur principal reste aujourd'hui Paul-Henri Antonmattei (qui a été un thésard de Bernard Teyssié).

La figure de Bernard Teyssié n'est bien entendu pas représentative de l'ensemble des travaillistes même si sa position centrale montre l'inclinaison du champ vers la droite de l'échiquier politique. On pourrait insister sur des individus orientés à gauche, et qui, dans les dernières années, ont pu 
contribuer, parfois à leur corps défendant, à la mise sur l'agenda de la nécessité de réformer le droit du travail. La figure d'Antoine Lyon-Caen en est un exemple : né la même année que Bernard Teyssié, fils de Gérard Lyon-Caen, il est professeur à Nanterre de 1979 à sa retraite en 2013. Il y dirige le DEA, devenu master, de droit social, ainsi que l'École doctorale de droit de 1992 à 1998. Il a dirigé lui aussi de très nombreuses thèses (certains de ses thésards sont maintenant en poste à Nanterre), et co-publié de nombreux manuels de droit du travail faisant autorité aujourd'hui encore. Sa position centrale vient aussi de son initiative de créer la Revue de droit du travail, qui se positionne très clairement dans un droit du travail tourné vers le travailleur. Il est enfin un proche de Robert Badinter, qu'il a suivi au ministère de la Justice entre 1982 et 1986. Et c'est avec lui qu'en 2016 il rédige le rapport sur la simplification nécessaire du droit du travail, qui était censée inspirer la loi El Khomri ${ }^{13}$. Au-delà d'Antoine Lyon-Caen, c'est une philosophie du droit du travail bien spécifique qui est défendue par cette gauche du droit du travail, et qui consiste à faire le lien entre « le nécessaire accroissement de la flexibilité et l'indispensable maintien du modèle social français. » (Lokiec \& Robin-Olivie, 2006) Cette position incontestablement fragile, qui a été longtemps tenable, devient impossible quand, en 2016-2017, c'est, comme on l'a vu, l'ensemble de l'architecture du droit du travail qui est mise en question.

\section{Acteurs et espaces de la production d'un droit du travail entrepreneurial}

Les espaces sociaux où se constitue ce nouveau droit du travail sont donc divers, et nous en avons jusqu'ici principalement évoqué deux : le champ politico-administratif et le champ académique. Revenir sur d'autres espaces, d'autres lieux de discussion et de production de normes et de discours, permet d'élargir la réflexion en introduisant l'analyse de «lieux neutres », au sens où les entendaient Luc Boltanski et Pierre Bourdieu : comités de rédaction de revues, conférences, cabinets ministériels, commissions... Toutes ces structures réunissent des spécialistes du droit du travail, et plus largement $\mathrm{du}$ « social», et elles sont « le lieu d'une circulation circulaire [des idées], propre à produire un effet d'auto-confirmation et d'auto-renforcement, et par là l'illusion de l'évidence immédiate » (Bourdieu \& Boltanski, 1976:61). Ces espaces contribuent à produire et à entretenir un sens commun de la réforme, en l'occurrence l'existence d'une seule manière de réformer le droit du travail, celle qui renforce la « sécurité » et la rentabilité des firmes au détriment de la protection des salariés.

Comme on l'a saisi dans l'analyse des réformes, on voit bien comment ce sens commun réunit des acteurs très divers, à la fois en termes d'appartenance professionnelle et de positionnement partisan, mais qui finalement partagent une même idéologie dominante concernant la nécessité de réformer, dans un sens entrepreneurial, le droit du travail. Les années 2012-2017 voient l'apogée de ces rapprochements divers, pendant le quinquennat Hollande et au début du quinquennat Macron. C'est donc sur ces années que nous nous focaliserons, même s'il est évident que ces proximités existent depuis bien plus longtemps. Ces rapprochements sont au moins de deux ordres. Le premier est donc d'ordre partisan, entre ce que l'on pourrait appeler le "centre-gauche », gagné au libéralisme, et la droite libérale. Les solutions préconisées et mises en œuvre par les uns et les autres ne diffèrent pas, et en ce sens, la continuité est parfaite par-delà les clivages officiels. Mais le second rapprochement renvoie à des restructurations plus profondes encore et matérialise le "grand brouillage » entre la sphère publique et les intérêts privés (France \& Vauchez, 2017). Les cabinets ministériels, mais aussi les commissions donnant lieu aux rapports que nous avons analysés ou encore les colloques de la revue Droit social, manifestent les passages, les allers-retours, les doubles rôles (parfois les doublesjeux) entre les mondes des avocats, des magistrats, des universitaires, des consultants et des grandes entreprises, en l'occurrence des DRH. D'une manière sans doute moindre que dans le droit des affaires, puisque les intérêts financiers y sont moins élevés, le droit du travail est un espace privilégié de ces circulations. On n'en donnera que deux exemples parmi tant d'autres, d'une part les positions politiques et professionnelles des conseillers «sociaux» des actuels cabinets présidentiel et

\footnotetext{
${ }^{13}$ Il faut noter qu'il s'opposera ensuite fermement à certaines mesures de la loi El Khomri et aux ordonnances
} Macron de 2017. 
ministériels, et d'autre part, d'une manière apparemment très différente, les colloques annuels de la revue Droit social ${ }^{14}$.

\section{Les acteurs politico-administratifs de la Loi Travail}

Une rapide biographie collective des collaborateurs de la première ministre du Travail d'Emmanuel Macron, Muriel Pénicaud, et des actuels conseillers sociaux de Matignon et de l'Élysée montre de manière saisissante ce brouillage professionnel et politique, mais aussi la continuité avec les précédentes équipes, ainsi que la matérialisation d'un monde du « social» que ces acteurs qualifient de "réformiste». Muriel Pénicaud cristallise l'ensemble de ces éléments. Issue du milieu de la bourgeoisie économique des Yvelines, elle entre dans l'administration du travail en 1985 et devient en 1991 membre du cabinet de Martine Aubry, alors ministre du Travail. Elle passe alors dans le privé, d'abord chez Danone jusqu'en 2002 puis chez Dassault System, avant de revenir chez Danone en 2008 au poste de directrice des ressources humaines. Elle multiplie dans le même temps les positions à michemin entre l'expertise et le politique autour des questions du travail (elle est par exemple membre du Haut conseil pour le dialogue social et chargée en 2010 par François Fillon, alors ministre du Travail, puis d'une mission sur le stress au travail).

Les autres conseillers sociaux des différents cabinets présidentiel et ministériels, bien que beaucoup plus jeunes, ont des parcours proches. C'est d'abord le cas en termes politiques : Pierre-André Imbert, conseiller «travail » du cabinet du Président de la République, a d'abord été le conseiller d'Henri Emmanuelli quand celui-ci était président socialiste de 1'Assemblée nationale entre 1997 et 2002, puis membre du cabinet des trois ministres du travail successifs du quinquennat Hollande (il était conseiller de Michel Sapin, puis directeur du cabinet de François Rebsamen et de Myriam El Khomri). Franck Morel, conseiller social d'Édouard Philippe, est lui proche de la droite, dans la mesure où il a été membre des cabinets des ministres du travail sous la présidence de Nicolas Sarkozy (Xavier Bertrand, Brice Hortefeux, Xavier Darcos et Éric Woerth).

Le «brouillage » des positions est aussi très visible sur le plan professionnel. Antoine Foucher, directeur de cabinet de Muriel Pénicaud, est d'abord administrateur du Sénat, avant de devenir conseiller de Laurence Parisot, la présidente du Medef, puis en 2016 directeur des relations sociales de Schneider Electric. Claire Scotton, directrice-adjointe du cabinet, est énarque, d'abord inspectrice des affaires sociales et adjointe au sous-directeur des relations individuelles et collectives de travail à la direction générale du travail. Après son passage au cabinet du ministère du travail entre 2017 et 2018 , elle entre chez Orange comme directrice de la prospective, stratégie et ressources humaines. Enfin, parmi de nombreux autres exemples, Franck Morel, déjà évoqué, commence sa carrière à l'inspection puis à l'Institut national du travail, de l'emploi et de la formation professionnelle, avant d'être recruté en 2011 comme avocat associé au cabinet Barthélémy. Ainsi, dans le domaine du droit du travail, on peut remarquer, comme le font Pierre France et Antoine Vauchez, « la convertibilité inédite, quoique circonscrite, des ressources et des titres acquis dans le service de l'État» (France \& Vauchez, 2017 : 47). Il est probable que cette convertibilité soit encore plus circonscrite dans le domaine du droit du travail que dans celui du droit public ou du droit des affaires, mais on voit qu'elle existe néanmoins.

Enfin, plusieurs membres des différents cabinets étudiés défendent une position particulière à l'endroit du «social», entendu en réalité comme le monde du travail et comme un intérêt particulier pour les négociations avec les organisations syndicales et patronales, et par conséquent pour le « dialogue social ». Le meilleur exemple est sans doute celui de Marc Ferracci, certes économiste très orthodoxe (il est professeur de sciences économiques à Paris 1 et affilié au CREST après avoir soutenu une thèse sous la direction de Pierre Cahuc, que nous avons déjà évoqué), mais aussi proche du social par son père. En effet, Pierre Ferracci, au départ expert-comptable, a fondé le groupe Alpha, société de conseil dont une des pierres angulaires est le cabinet Secafi, cabinet d'expertises à destination des anciens CHSCT et proche de la CGT $^{15}$. De même, Pierre-André Imbert a eu des postes de direction

\footnotetext{
${ }^{14}$ Il aurait par exemple été très intéressant d'étudier la composition des commissions produisant les différents rapports sur la réforme du droit du travail : sur la commission Combrexelle de 2015, voir Légé, 2019.

${ }^{15}$ On peut noter pour l'anecdote qu'il est aussi président du Paris Football Club (PFC), actuel pensionnaire du
} 
chez Altedia puis chez Alixio, les cabinets en ressources humaines fondés et dirigés par Raymond Soubie ${ }^{16}$. À l'instar d'Antoine Foucher, qui déclare en septembre 2017 : «j'ai toujours tourné autour du social, c'est ce que $\mathrm{j}$ 'avais dans les tripes ${ }^{17}{ }^{17}$, nombre de ces conseillers font toute leur carrière dans le public mais aussi dans l'entreprise, dans les relations sociales, les ressources humaines, le droit social, etc.

\section{Les colloques de la revue Droit social}

Tous les ans a lieu le colloque de la revue Droit social, animé par Jean-Emmanuel Ray, et qui réunit, autour d'une thématique spécifique, universitaires et praticiens du droit du travail. La revue Droit social est depuis sa naissance en 1938 un lieu de publication de la recherche en droit du travail, mais aussi de mise en forme d'une position que nous avons appelée ailleurs "centrale et centriste " (Willemez, 2017 : 80-82). Institutionnaliste à son origine, la revue créée par Paul Durand, dont le credo était de n'être ni marxiste ni libérale, a relativement évolué, et elle est aujourd'hui dirigée par Christophe Radé, professeur à Bordeaux. Même si elle est centrale dans la discipline, et a par conséquent vocation à accueillir l'ensemble des positions possibles, elle organise des colloques qui, pour leur part, défendent assez clairement un droit du travail en phase avec le tournant entrepreneurial. Il n'est qu'à lire le thème de ses dernières éditions : celui de 2015 était consacré à « Droit du travail et qualité de vie au travail », la qualité de vie au travail (QVT) étant désormais, pour résumer en quelques mots, la traduction managériale et déconflictualisée de la thématique des conditions de travail en entreprise (Levet, 2013). Le colloque de 2018 était lui consacré à la question suivante : « Quelle place pour les syndicats dans le nouveau modèle social? », et relatif à la mise en application des ordonnances Travail de 2017.

Dans le cadre de ce dernier colloque, c'est surtout le «casting» des intervenants qui interpelle et montre à la fois la perspective politique désormais suivie et le type d'alliance que les animateurs du colloque et de la revue constituent. Seuls deux universitaires interviennent, situés des deux côtés du champ politique : Patrick Lokiec, qui défend donc un droit du travail protecteur pour les salariés, et Pierre-Henri Antonmattei, qui est lui dans la promotion d'un droit du travail entrepreneurial. Les autres intervenants sont tous situés en dehors du champ académique : des avocats (là aussi, l'équilibre est respecté : la première dans un cabinet spécialisé dans la défense des salariés, et le second dans un cabinet employeurs), des cadres dirigeants d'entreprises (en ressources humaines mais pas seulement), et des syndicalistes ou d'anciens syndicalistes reconvertis dans le conseil en relations sociales. Parmi ces derniers, on note la présence de Marcel Grignard, ancien secrétaire général adjoint de la CFDT (de 2009 à 2014), devenu président de «Confrontations Europe », qui se définit ainsi sur son site Internet : «Confrontations Europe, un think tank européen, un réseau, un lobby d'intérêt général. Partager des diagnostics et élaborer des propositions concrètes pour mieux construire une Europe humaniste, compétitive et solidaire ${ }^{18}$. Pour ce qui nous intéresse ici, Marcel Grignard est devenu co-président du «comité d'évaluation des ordonnances travail» avec son homologue, Jean-François Pillard, professeur à ESCP Europe, qui semble, lui, représenter la partie patronale et est, lui aussi, présent au colloque. Une dernière table-ronde réunit des cadres dirigeants d'entreprise (notamment un chef de projet digital et le directeur des relations sociales de Solvay) et Franca Salis-Maliniera, secrétaire nationale de la CFDT. On voit ainsi le clivage que le colloque tente de produire, par-delà la présence de Patrick Lokiec et de Judith Krivine: les différences entre les parties sont minimes, et ce qui compte, c'est l'accord général sur la nécessité de favoriser la bonne santé économique des entreprises et l'attention qui doit néanmoins être portée aux salariés par l'intermédiaire de leurs représentants « réformistes » et ouverts au « dialogue ».

championnat de Ligue 2 !

${ }^{16}$ Raymond Soubie a une très longue carrière de conseiller en matière de travail : commencée en 1969 dans le cabinet de Joseph Fontanet, ministre du Travail de Georges Pompidou, elle se termine à l'Élysée, puisqu'il est conseiller social de Nicolas Sarkozy de 2007 à 2012.

${ }^{17}$ Beloezzane Sarah et Bissuel Bertrand, «Au cœur de la fabrique des ordonnances réformant le code du travail », Le Monde, 22 septembre 2017, mis à jour le 23 septembre 2017.

${ }^{18}$ Voir le site : http://confrontations.org/ (consulté le 01/12/2020). 
Le droit du travail a donc été profondément transformé. Ce n'est alors pas sa disparition qu'il faut acter mais le profond changement de sens dont il est l'objet, une véritable " révolution symbolique », dans la mesure où c'est sa légitimité sociale et la manière de le penser intellectuellement et dans l'action qui sont totalement modifiées. C'est ce qui produit l'impression que les alternatives sont impensables ou du moins inaudibles. Celles-ci existent pourtant. Un certain nombre d'universitaires organisent des colloques et publient des travaux critiques sur les ordonnances Macron. De même, l'initiative portée par Emmanuel Dockès autour de la réécriture du Code du travail a eu un certain écho, au sein du champ académique et au dehors. Dans d'autres espaces sociaux, la CGT et ses avocats proches poursuivent leur travail de soutien des salariés, lors des licenciements collectifs et aux prud'hommes. Pour cette dernière arène, ils vont d'ailleurs plus loin et utilisent les outils juridiques pour remettre en cause certains aspects des dernières réformes (Willemez, 2003), comme on a pu le voir lors des dernières décisions des conseils de prud'hommes allant dans leurs jugements au-delà du plafonnement des indemnités imposé par l'une des ordonnances au nom de la Charte sociale européenne et de la Convention de l'Organisation internationale du travail (OIT).

C'est dire que les initiatives ne manquent pas. En l'absence de perspective claire de mobilisation forte sur le droit du travail ou de véritable alternance politique, ce sont d'autres voies qui sont imaginées ou relancées, et qui doivent permettre de faire du droit du travail ce qu'il a longtemps été et ce pour quoi il a été inventé : un cadre de protection des travailleurs face à l'arbitraire patronal.

\section{Références bibliographiques}

AMABle Bruno \& PAlOMBARINI Stefano, 2018. L'Illusion du bloc bourgeois : alliances sociales et avenir du modèle français, Paris, Raisons d'Agir.

BARTHELEMY Jacques \& CETTE Gilbert, 2015. Réformer le droit du travail, Paris, Odile Jacob.

BEHRENT Michael C. 2010. «Accidents Happen: François Ewald, the "Antirevolutionary" Foucault, and the Intellectual Politics of the French Welfare State », The Journal of Modern History [En ligne], 82 (3), p. 585-624. Consulté le 28/11/2020. URL : www.jstor.org/stable/10.1086/653042 ; DOI : $10.1086 / 653042$

BILAND Émilie, 2013. «Quand les managers mettent la robe. Les grandes écoles de commerce sur le marché de la formation juridique », Droit et société [En ligne], 83, p. 49-65. Mis en ligne le 21/05/2013 (consulté le 28/11/2020). URL: https://www.cairn-int.info/revue-droit-et-societe12013-1-page-49.htm ; DOI : 10.3917/drs.083.0049

BOISSONNAT Jean, 1995. Le Travail dans vingt ans, Paris, Odile Jacob/La Documentation française.

BOURDIEU Pierre, 1984. Homo academicus, Paris, Éditions de Minuit.

—, 2013. Manet. Une Révolution symbolique, Paris, Raisons d'Agir-Seuil.

BOURDIEU Pierre \& BOLTANSKI Luc, 1976. «La production de l'idéologie dominante », Actes de la recherche en sciences sociales [En ligne], 2, p. 3-73. Consulté le 28/11/2020. URL :

www.persee.fr/doc/arss 0335-5322 1976 num 223443 ; DOI : 10.3406/arss.1976.3443

CAHUC Pierre \& KRAMARZ Francis, 2004. De la Précarité à la mobilité : vers une sécurité sociale professionnelle : rapport au Ministre de l'économie, des finances et de l'industrie et au Ministre de l'emploi, du travail et de la cohésion sociale, Paris, La Documentation française.

CHAPOULIE Jean-Michel, 2001. La Tradition sociologique de Chicago : 1892-1961, Paris, Éditions du Seuil.

CHARLE Christophe \& SOUlie Charles (dir.), 2007. Les Ravages de la "modernisation » universitaire en Europe, Paris, Syllepse.

DARDOT Pierre \& LAVAL Christian, 2009. La Nouvelle raison du monde: essai sur la société néolibérale, Paris, La Découverte.

DENORD François, 2016. Le Néo-libéralisme à la française: histoire d'une idéologie politique, Marseille, Agone.

DIDRY Claude, 2016. L'Institution du travail : droit et salariat dans l'histoire, Paris, La Dispute.

EWALD François, 1983. Le Droit du travail : une légalité sans droit?, Paris, Fondation Saint-Simon.

-, 1986. L'État providence, Paris, Grasset.

FRANCE Pierre \& VAUCHEZ Antoine. 2017. Sphère publique, intérêts privés : enquête sur un grand brouillage, Paris, Presses de Sciences Po. 
GUILBERT Thierry, 2008. Le Discours idéologique ou La force de l'évidence, Paris, L'Harmattan.

GUILBERT Thierry, LEBARON Frédéric \& PEÑAFIEL Ricardo. 2019. "Introduction. Discours austéritaires et discours néolibéral », Langage et société [En ligne], 166, p. 9-29. Mis en ligne le 21/01/2019 (consulté le 28/11/2020). URL : https://www.cairn.info/revue-langage-et-societe-20191-page-9.htm ; DOI : 10.3917/ls.166.0009

JUHEM Philippe, 2001. "La légitimation de la cause humanitaire: un discours sans adversaires », Mots. Les langages du politique [En ligne], 65, p. 9-27. Consulté le 28/11/2020. URL: https://www.persee.fr/doc/mots 0243-6450 2001 num 6512484 ; $10.3406 /$ mots.2001.2484

LE GOFF Jacques, 2004. Du Silence à la parole : une histoire du droit du travail, Presses universitaires de Rennes.

LEGE Philippe, 2019. Production et légitimation d'une réforme : le "projet de loi Travail », Vulainessur-Seine, Éditions du Croquant.

LEVET Pascale, 2013. «Des risques psychosociaux à la Qualité de Vie au Travail », Négociations [En ligne], 19 (1), p. 97-111. Mis en ligne le 05/06/2013 (consulté le 28/11/2020). URL: https://www.cairn.info/revue-negociations-2013-1-page-97.htm ; DOI : 10.3917/neg.019.0097

LOKIEC Pascal \& ROBIN-OLIVIE Sophie, 2006. «Le discours sur la flexibilité, le droit du travail et l'emploi », Revue de droit du travail, 1, p. 48-63.

Mias Arnaud, Guillaume Cécile, Denis Jean-Michel \& Bouffartigue Paul, 2016. «Vers un "dialogue social" administré? », La nouvelle revue du travail [En ligne], 8. Mis en ligne le 31/05/2016 (consulté le 28/11/2020). URL : http://journals.openedition.org/nrt/2560; DOI : $10.4000 /$ nrt. 2560

TEYSSIE Bernard, 2005. «Sur l'entreprise et le droit du travail : prolégomènes », Droit social, 2, p. $127-128$.

TRACOL Matthieu, 2009. Changer le travail pour changer la vie: genèse des lois Auroux, 1981-1982, Paris, L'Harmattan.

WILLEMEZ Laurent, 2003. "Quand les syndicats se saisissent du droit», Sociétés contemporaines [En ligne], 52, p. 17-38. Consulté le 28/11/2020. URL: https://www.cairn.info/journal-societescontemporaines-2003-4-page-17.htm ; DOI : 10.3917/soco.052.0017

-, 2017. Le Travail dans son droit. Sociologie historique du droit du travail en France (1892-2017), Paris, LGDJ. 